Panel A. Forest Plot of correlation of measured HCQ blood levels with assessed adherence

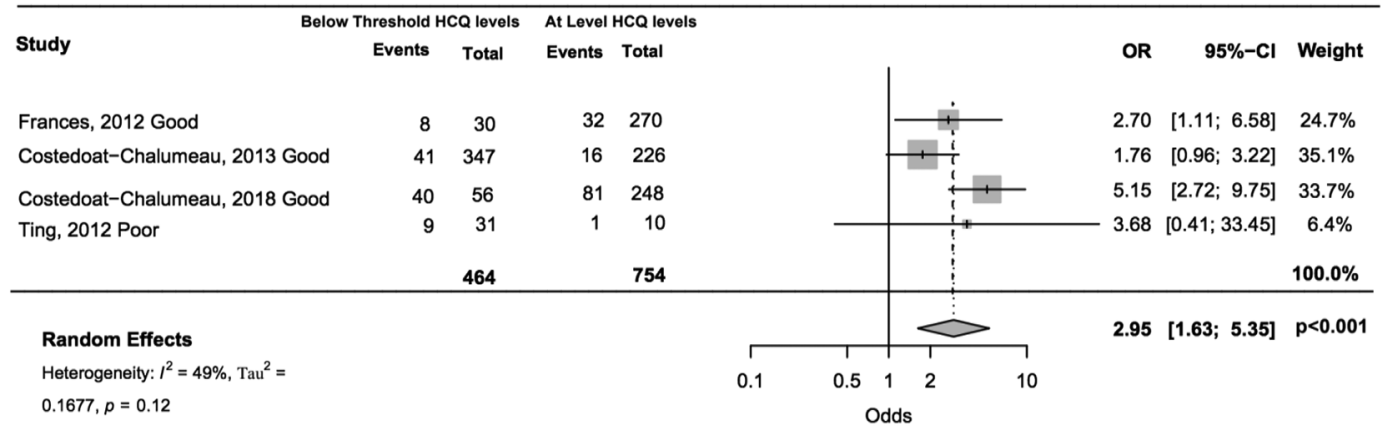

Panel B. Forest Plot of correlation of measured HCQ levels with SLEDAI scores

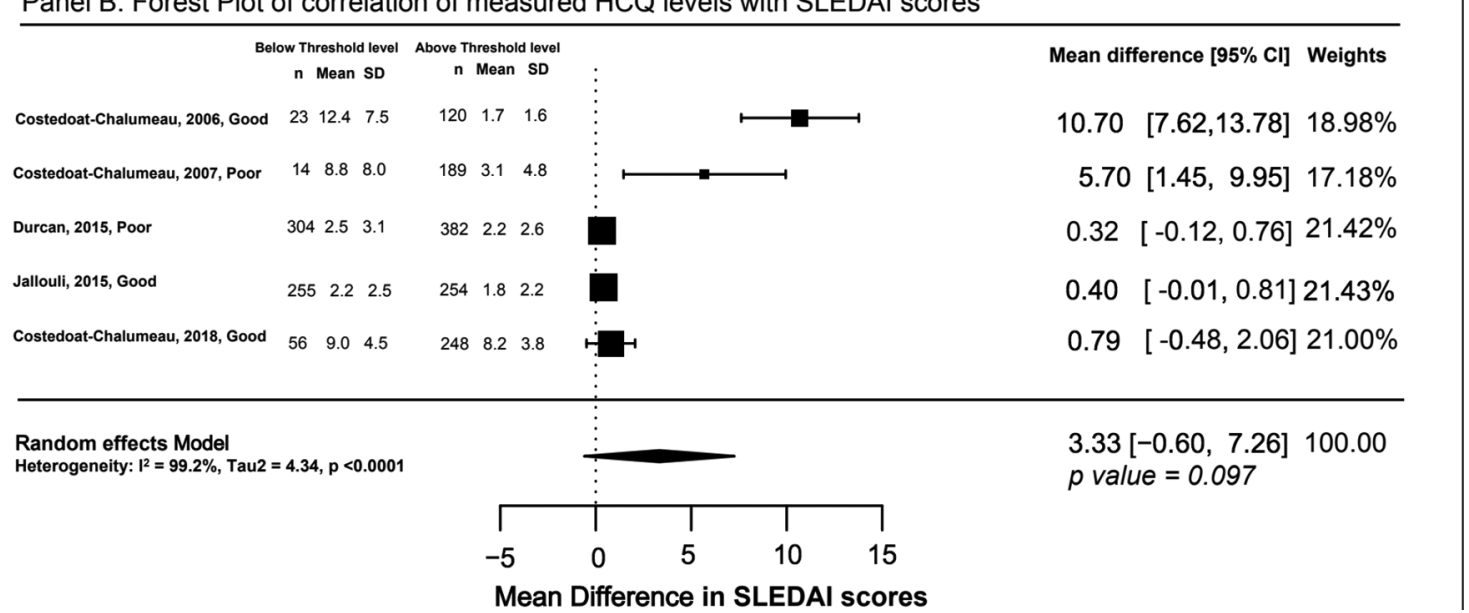

Abstract 221 Figure 1 Forest plot showing correlation of HCQ blood levels with A) Assessed Adherence B) SLEDAI scores

SLEDAI scores but due to the limited number of studies and heterogeneity we did not find significant correlation. Findings support the clinical utility of measuring HCQ levels in SLE patients. Future larger studies should investigate standards for HCQ level interpretation and target levels needed to minimize SLEDAI scores.

Funding Source(s): n/a

\section{CIRCULATING PENTRAXIN3-SPECIFIC B CELLS ARE DECREASED IN LUPUS NEPHRITIS}

${ }^{1}$ Mariele Gatto*, ${ }^{2}$ Annika Wiedemann, ${ }^{2}$ Nadja Nomovi, ${ }^{2}$ Karin Reiter, ${ }^{3}$ Eva Schrezenmeier, ${ }^{2}$ Thomas Rose, ${ }^{2}$ Franziska Szelinski, ${ }^{4}$ Andreia Lino, ${ }^{5}$ Sonia Valentino, ${ }^{6}$ Anna Ghirardello, ${ }^{7}$ Thomas Dörner, ${ }^{1}$ Andrea Doria. ${ }^{1}$ University of Padova, Department of Medicine, Division of Rheumatology; ${ }^{2}$ Department Medicine/Rheumatology and Clinical Immunology Charite' Universitätsmedizin Berlin, Germany; ${ }^{3}$ Department of Medicine/Nephrology and Medical Intensive Care Charité - Universitätsmedizin Berlin, Charitéplatz 1, 10117 Berlin; ${ }^{4}$ Deutsches Rheuma-Forschungszentrum, A Leibniz Institute, Berlin, Germany; ${ }^{5}$ Humanitas Clinical and Research Center, Milan, Italy; ${ }^{6}$ Unit of Rheumatology, Department of Medicine, University of Padova, Padova, Italy; ${ }^{7}$ Charite Universitätsmedizin Berlin and Deutsches Rheuma-Forschungszentrum (DRFZ)

10.1136/lupus-2019-Ism.222
Background Pentraxin3 (PTX3) is overexpressed in kidneys of patients developing lupus nephritis (LN). Active LN is associated with reduced anti-PTX3 antibodies. However, abnormalities of B cell differentiation against PTX3 have not been characterized in systemic lupus erythematosus (SLE).

The aim of our study is to characterize PTX3-specific $($ PTX3 +$)$ B cells in peripheral blood of SLE patients with or without LN and healthy donors (HD).

Methods SLE patients without LN, biopsy-proven LN and matched $\mathrm{HD}$ were analyzed. Active $\mathrm{LN}$ was defined as proteinuria $>0.5 \mathrm{~g} /$ day or $\mathrm{CrCl}<60 \mathrm{ml} / \mathrm{min} / 1.73 \mathrm{~m}^{2}$ with active urinary sediment. Peripheral B cells were analyzed for direct PTX3 binding by flow cytometry using PTX3 labeled with cyanine 5 (Cy5) and phycoerythrin (PE).

Results Initially, a flow cytometry based assay to identify PTX3 + B cells was developed by demonstrating simultaneous binding of PTX3-Cy5 and PTX3-PE. Specificity of B cells was validated by blocking experiments using unlabeled PTX3 (figure 1). We could identify circulating PTX $3+\mathrm{B}$ cells in HD and patients. Notably, LN patients showed a significantly diminished number of PTX3 +B cells (SLE vs. LN p=0.033; HD vs. LN $p=0.008$ ). This decrease was identified in naïve and memory $B$ cell compartments (naïve: SLE vs. LN 
A
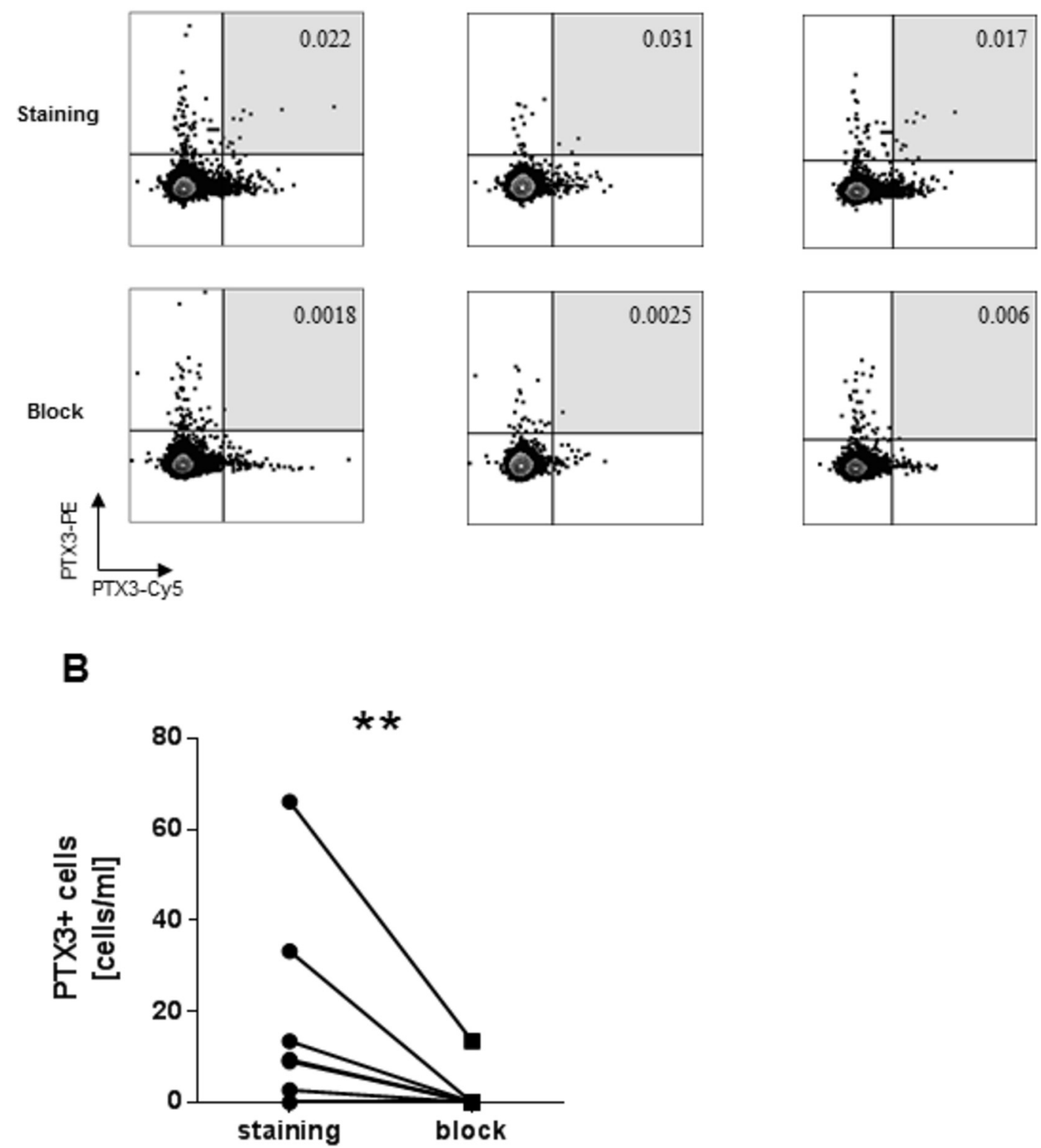

Abstract 222 Figure 1 Identification of PTX3+ B cells among CD19+CD20+ B cells. (A) Three representative dot plots of the PTX3-specific B cells before and after blocking with unlabeled PTX3. Only B cells staining positive for both PTX3-Cy5 and PTX3-PE were considered (light gray square). (B) Quantification of PTX3 biding among B cells before and after blocking of PTX3. Footnotes: Cy5, cyanin 5; PE, phycoerythrin

$\mathrm{p}=0.028 ; \mathrm{HD}$ vs. LN $\mathrm{p}=0.0001 ;$ memory: SLE vs. $\mathrm{LN}$ $\mathrm{p}=0.038$, HD vs. $\mathrm{LN} \mathrm{p}=0.011$ ) (figure 2).

Conclusions Decreased PTX3 +B cells in LN within the naïve and memory compartment suggest their negative selection at early stages of B cell development potentially related to a decreased regulatory function. PTX3 $+\mathrm{B}$ cells could candidate for autoantigen-defined regulatory $\mathrm{B}$ cells as a striking abnormality of LN patients 
A
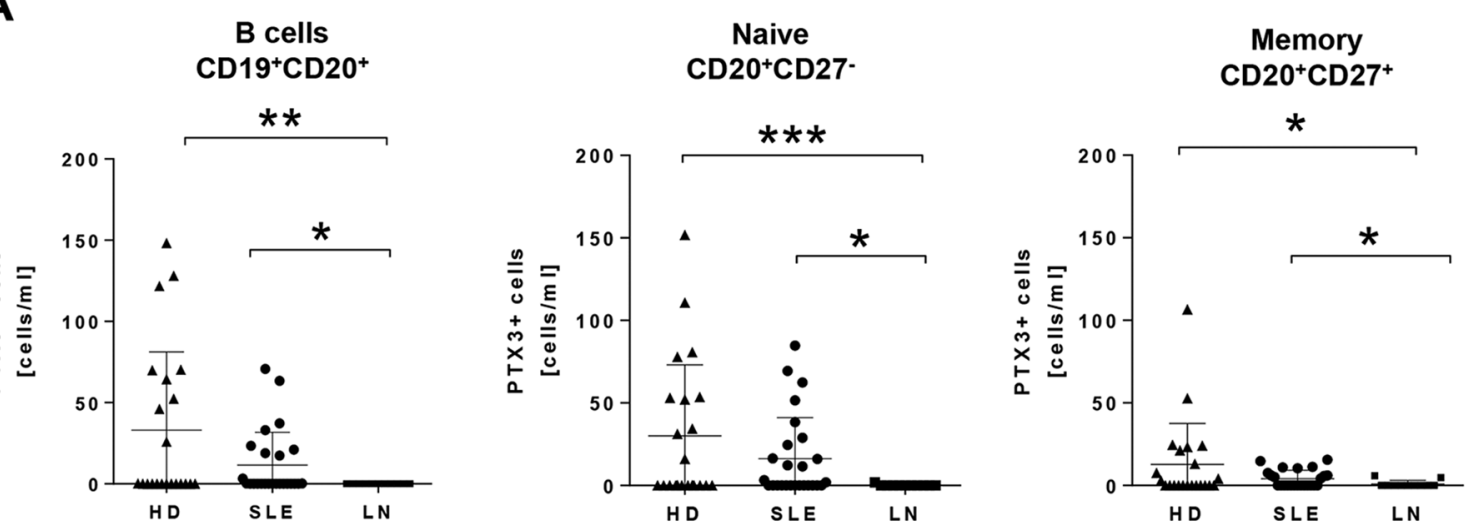

B
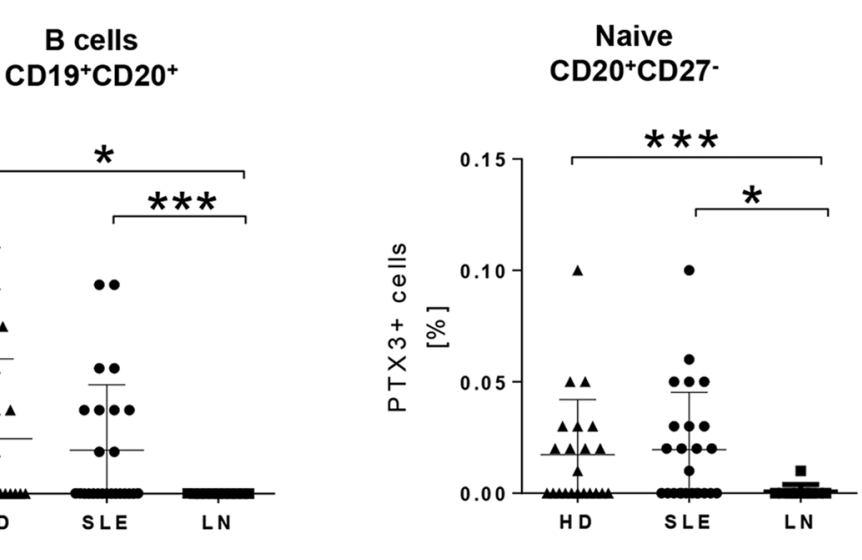

C

HD

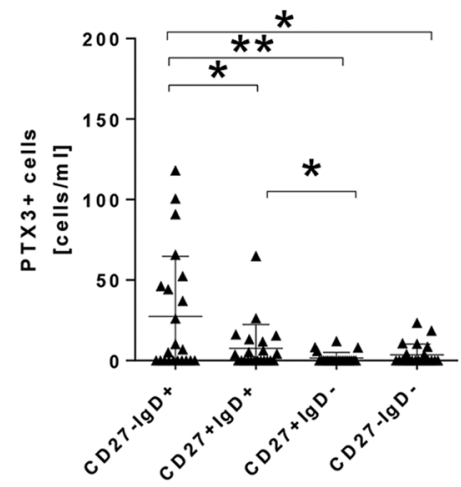

D

HD

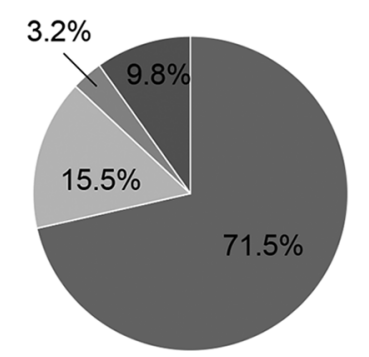

SLE

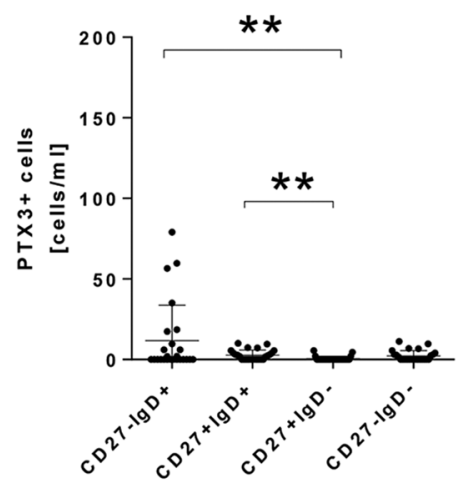

SLE

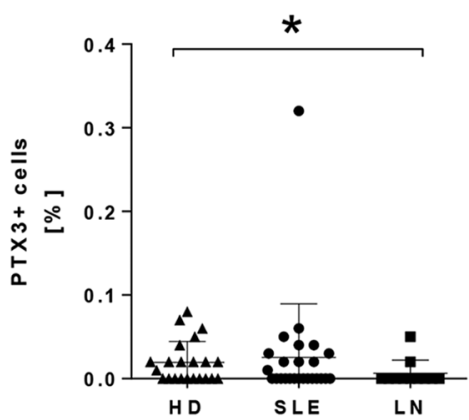

LN

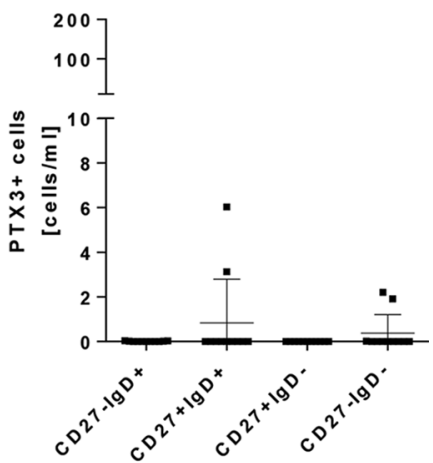

LN
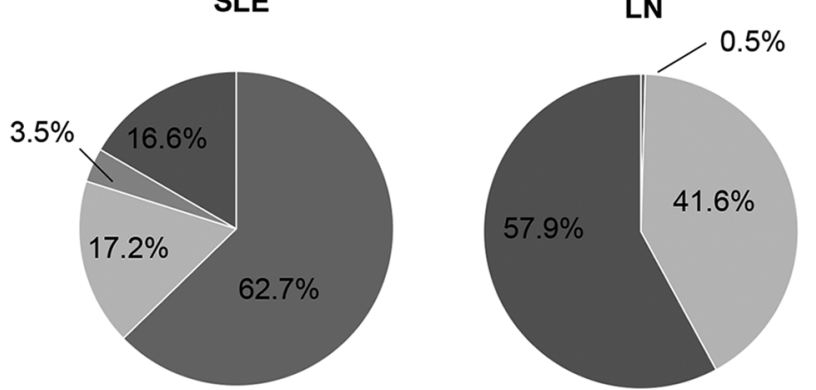

- $\mathrm{PTX3+CD27-lgD+}$

- $\mathrm{PTX} 3+\mathrm{CD} 27+\operatorname{lgD}+$

- PTX3+CD27+lgD-

- PTX3+CD27-IgD-

Abstract 222 Figure 2 PTX3+ B cells are decreased in patients with lupus nephritis and are mainly confined to CD27-lgD+ B cells. (A) Absolute numbers of PTX3+B cells (cell/mL) within left) total; middle) naïve or right) memory B cells in HD $(n=22)$ and SLE $(n=26)$ and $L N(n=12)$ patients. (B) Frequencies of PTX3+ B cells (left), naïve (middle) and memory (right) are decreased in $L N(n=12)$ in comparison with HD ( $n=22)$ and SLE ( $n=26)$. (C) Distribution of CD27 and IgD expression by PTX3+ B cell subsets are shown. Enrichment in the naïve pool with decreases in the other subsets was found in HD $(n=22)$ and SLE $(n=26)$, but not in LN $(n=12)$. (D) Pie charts of percentages of PTX3+ CD27lgD subsets within the PTX3+ B cell pool are consistent with distribution of absolute numbers. Mann-Whitney $\mathrm{U}$ test. $\left({ }^{*}<0.05,{ }^{* *}<0.01,{ }^{* *}<0.001\right)$. Footnotes: SLE, systemic lupus erythematosus; HD, healthy donors; LN, lupus nephritis; PTX3 pentraxin3 